\title{
Qualitative Descriptors Used by Patients Following Orthognathic Surgery to Portray Altered Sensation
}

\author{
Ceib Phillips, MPH, PhD*, Greg Essick, DDS, PhD ${ }^{\dagger}$, John Zuniga, DMD, PhD $\ddagger$, Myron Tucker, \\ DDS§, and George Blakey III, DDS\| \\ Department of Orthodontics, University of North Carolina School of Dentistry, Chapel Hill, NC.
}

\begin{abstract}
Purpose-Following orthognathic surgery, patients use qualitatively different words to describe the altered sensation on their face that results from tissue inflammation and nerve injury. These words indicate normal, hypoesthetic, paresthetic, and dysesthetic sensations, and reflect the intrusiveness of the alteration. Our intent was to study the words chosen by patients from a standardized list to characterize sensory recovery during the first 6 months after surgery and to examine whether patients who underwent different surgical procedures tended to choose different sets of words.
\end{abstract}

Patients and Methods-Patients' selections from a list of 27 words that described their assessment of spontaneous and evoked facial sensations were obtained before surgery and at 1 week, 1 month, 3 months, and 6 months after surgery. Data were obtained from 146 patients enrolled in a randomized controlled clinical trial designed to evaluate the potential of sensory retraining in the rehabilitation of patients who experience impairment in sensory function after nerve injury. Mantel Haenszel general correlation and row mean score statistics were used to assess the association between time and word choice and to compare the word choice categories of 4 surgical groups: bilateral sagittal split osteotomy (BSSO) only, with or without genioplasty; BSSO + Le Fort I, with or without genioplasty.

Results-In general, the number of words selected to describe the alteration in sensation decreased over time, as did the intrusiveness of the category of words chosen. However, the intrusiveness remained the same or worsened from 1 week to 6 months for $32 \%$ of patients. With increased time after surgery, the percentage of patients who reported altered evoked sensations exceeded the percentage who reported spontaneous sensations. For example, at 6 months the altered sensation of $66 \%$ of the patients was classified in the paresthesia and dysesthesia categories by the evoked assessment of sensation; whereas, that of only $47 \%$ of the patients were classified as such by the spontaneous assessment. The addition of Le Fort I to BSSO did not affect the way patients reported altered sensation on their lower face. Hypoesthesia and paresthesia, but not dysesthesia, were less of a problem on the midface than on the lower face after BSSO + Le Fort I. Patients who had genioplasty more frequently chose descriptors for the lower face that reflected soft tissue trauma and inflammation ("swollen," "tender," and "burning") than patients without genioplasty; however, this difference decreased with time after surgery.

\footnotetext{
(C) 2006 American Association of Oral and Maxillofacial Surgeons

Address correspondence and reprint requests to Dr Phillips: Department of Orthodontics, University of North Carolina School of Dentistry, CB 7450, Chapel Hill, NC 27599; ceib_phillips@ dentistry.unc.edu.

*Professor.

†Professor.

†Professor.

\$Private Practitioner.

"Clinical Assistant Professor.
} 
Conclusion-The current findings indicate that patients' selection of words differentiates individuals who experienced only a simple loss in sensation (ie, present negative symptoms), those who experienced active sensations that are not normally present (ie, present positive symptoms), and those whose active sensations are additionally uncomfortable or painful. It is possible that continued study of the latter group of patients will reveal patterns of word usage that predict poor long-term recovery and disabling sensory disorders.

Osteotomies used in orthognathic surgery are performed in the vicinity of sensory nerves of the maxillary and mandibular divisions of the trigeminal nerve. Transient altered sensation over their cutaneous distributions always occurs following surgery because of soft tissue edema and inflammation, or direct or indirect injury to the nerves. ${ }^{1,2}$ The rate and extent at which facial sensation recovers are affected by the type of surgery performed, the number of procedures performed in proximity to the same division of the trigeminal nerve, and biological factors such as patient age. ${ }^{1,3-10}$

Early investigators of complications following orthognathic surgery emphasized the importance of objective measures of sensory function for the detection and characterization of nerve injury. ${ }^{3,5}, 11$ However, most studies conducted over the past 20 years have found that neither the incidence nor complexity of patients' subjective impairment is captured by abnormal results on an individual neurosensory test.6. $12^{-14}$ Recent work has indicated that the highest sensitivity and specificity in detecting the impairment reported by patients (or identified by specialized nerve conduction studies) is found when the patient's performance is "abnormal" on any quantitative sensory test of tactile and thermal sensory function. ${ }^{14-} 18$

Indeed, in a clinical setting, the patient's recognition that an alteration exists and his/her description of that alteration is essential for counseling of the patient, determining further testing, and deciding on treatment. However, investigations on patient-based "subjective" evaluations have been quite limited in the scope of the response options provided patients: for example, yes or no19 or a few verbal descriptors primarily focused on reduced sensitivity or numbness. $20^{-} 23$ Little work has focused on characterizing patients' verbal descriptions of altered sensation even though the latter has been suggested for the routine clinical evaluation of patients. ${ }^{24}$ Some patients report only diminished sensitivity (ie, negative symptoms such as numbness or dullness) implying loss of innervation; while others report tingling or pins-andneedles (ie, positive symptoms) implying neural sensitization. ${ }^{10,25}, 26$ Moreover, a small yet significant percentage of patients report spontaneous or stimulus-evoked pain, which may be refractory to contemporary treatments.

The implications of the differences in the choice of qualitative descriptors used by patients to report altered sensation are unknown: for example, do patients with qualitatively different reports of altered sensation (eg, numbness vs tingling vs burning) in the short term after surgery recover at a different rate or does the quality of the alteration have an effect on the patient's quality of life during recovery? Is it possible to distinguish alterations in sensation that result from nerve injury from those that result acutely from tissue trauma and inflammation? Do patients who undergo different types of orthognathic surgery (eg, surgery on the mandible and maxilla vs surgery on the mandible only) or more than 1 procedure on the same jaw (eg, genioplasty in addition to bilateral sagittal split osteotomy [BSSO] vs BSSO alone) experience qualitatively different altered sensations?

To better understand these issues, we investigated the words chosen by patients to describe altered sensation on their face. Based on the patients' selection of words, sensation on the face was classified and ranked in increasing order of intrusiveness as normal, hypoesthetic, paresthetic, or dysesthetic. The percentages of patients classified in the different categories and the extent of intrusiveness as represented by the word choices were analyzed to characterize 
sensory recovery during the 6-month period following surgery and to identify differences in the words chosen by patients who underwent different surgical procedures.

\section{Methods SUBJECTS}

Potential subjects were screened at the Oral and Maxillofacial Clinic at the University of North Carolina at Chapel Hill or at University Oral Maxillofacial Surgery in Charlotte, NC, a community-based practice. A limited waiver of HIPAA authorization was requested and received from the Institutional Review Board. Consecutive patients with surgery dates after December 1,2002 were eligible to be enrolled if they were scheduled for a BSSO alone or with Le Fort I (LFI) osteotomy, to correct a severe malocclusion and/or a developmental disharmony. Inclusion and exclusion criteria are provided in Table 1. Before a subject was enrolled, written consent (and assent if the subject was younger than 18) was obtained in accordance with the policies of the University of North Carolina at Chapel Hill School of Dentistry's Institutional Review Board. Each subject enrolled and consented after April 14, 2003 signed a HIPAA consent form as well. Surgeries were performed by 5 attending surgeons. Resident assistants were present during all surgeries.

All participants were enrolled in a multicenter, double-blind, 2-arm parallel group, stratified block randomized controlled clinical trial (Table 2). The clinical trial was designed to evaluate sensory retraining, a rehabilitative therapy that offers significant potential for patients who experience impaired sensory function. ${ }^{26,27}$ Half of the subjects were randomized to receive instruction on standard opening exercises only after surgery while the other half were also given a progressive series of sensory retraining exercises during which the patient systematically touched or stroked the face with a cosmetic brush. The altered sensation commonly caused by orthognathic surgery served as the experimental model. This report provides an exploratory analysis of the words chosen by patients to describe altered facial sensation.

\section{PROCEDURES}

Before surgery and at 1 week, 1 month, 3 months, and 6 months after surgery, subjects were questioned about altered sensation at 4 extraoral locations: the hairy upper lip, upper vermillion, lower vermillion, and chin. Initially, the patient was instructed to keep the face at rest and not make any facial expression. Using a mirror to aid location of each site, the patient was asked whether any altered sensation was being experienced at that site (spontaneous alteration). If altered sensation was present, the patient was then asked to choose at least 1 word from a standardized list (Table 3 ) to describe the altered sensation. ${ }^{24}$ More than 1 word could be selected for each site; 27 words were possible.

The entire procedure was then repeated, but the patient was asked to make facial expressions and to touch or rub each test site with a finger before responding (evoked alteration). These 2 assessments provide different but complimentary subjective information: alteration that occurs without any external influence (spontaneous) versus self- or stimulus-induced alteration (evoked).6,24 The evoked assessment is intended to evaluate sensation during simulated facial expressions and oral behaviors without tactile stimulation provided by the examiner (eg, during sensory testing). The 2 sets of chosen words characterized the spontaneous altered sensation and the evoked altered sensation, respectively, experienced by the patient. Patients who selected no words were categorized as having no altered sensation (normal sensation).

After consultation with 7 oral and maxillofacial surgeons, the words on the list were categorized as consistent with hypoesthetic, paresthetic, or dysesthetic sensations (Table 4).10,24,25 Words 
associated with hypoesthesia referred to a simple loss in sensation (eg, numbness), or relatively passive or benign alterations in sensation such as rubbery, swollen, warm, and wet. Words associated with paresthesia referred to active sensations that are not normally present, such as tingling, tickling, and pulling. Words associated with dysesthesia differed from those associated with paresthesia in that discomfort or pain was implied (eg, words such as tender, burning, and sore were in the dysesthesia category). The categories were rank ordered and scored according to the level of the intrusiveness of altered sensation $(I A S)$ : no alteration (value $=0)<$ hypoesthesia $($ value $=1)<$ paresthesia $($ value $=2)<$ dysesthesia $($ value $=3)$. Each facial site was scored by the most intrusive category from which words were selected for that site, providing values for $I A S_{\text {spon }}$ and $I A S_{\text {evok }}$ for each site. In addition, the most intrusive category from which word(s) had been chosen to describe any of the 4 sites was determined separately for the spontaneous and evoked alterations. IAS $S_{\max \text { spon }}$ and $I A S_{\max \text { evok }}$ defined the maximum intrusiveness of the altered sensation over the individual patient's face for the spontaneous and evoked assessments respectively. And finally, $I A S_{\max }$ defined the most intrusive category selected for either the spontaneous or evoked assessments.

\section{STATISTICAL ANALYSIS}

Analyses were performed using the intrusiveness score at each visit that represented the maximum intrusiveness reported at any of the 4 facial sites ( $I A S_{\max \text { spon }}$ and $\left.I A S_{\max \text { evok }}\right)$. Because both $I A S_{\max \text { spon }}$ and $I A S_{\max \text { evok }}$ responses were ordinally based (scored 0 to 3 ), stratified-by-subject repeated measures Mantel Haenszel correlation statistics were calculated to assess the association between time and the intrusiveness of altered sensation. A sensitivity analysis performed using modified ridits and different sets of scores $(0,1,3,5)$ and $(0,1,2,4)$ for the word categories indicated that the score assignment did not substantially change the conclusions. To assess whether the qualitative description at the 4 facial sites differed by type of surgery, Mantel Haenszel row mean score statistics were calculated to compare the average intrusiveness score of patients who underwent BSSO only or BSSO + LFI, with and without genioplasty at 1 week and 6 months. Analyses were performed separately for $I A S_{\max \text { spon }}$ and $I A S_{\text {max evok. }}$ Level of significance was set at .05 .

\section{Results}

The majority of the 146 participants were Caucasian and female (Table 2). All 146 patients underwent surgery in the vicinity of sensory nerves associated with the mandibular division, but only $61(42 \%)$ underwent surgery in the vicinity of the sensory nerves associated with the maxillary division. Patients who had a BSSO with a genioplasty were slightly younger (mean, 21.2 years) and those who had BSSO + LFI and a genioplasty were slightly older (mean, 27.4 years) than patients who did not have a genioplasty (Table 2).

\section{PATIENTS' SELECTION OF WORDS ACROSS CATEGORIES OF INTRUSIVENESS}

At 1 week after surgery the median number of words chosen to describe the sensation(s) that occurred spontaneously or were evoked during assessment across all 4 sites on the face was 9 and 7 , respectively. Twenty-five percent of the patients chose 14 or more words to describe the spontaneous alteration and 11 or more for the evoked alterations. By 6 months after surgery, the median number of words chosen by a patient for all sites decreased to 2 and 3 words, respectively. The seventy-fifth percentile value was 4 words for the spontaneous and 6 for the evoked assessments of sensation. This general trend was observed for each site individually. For example, for the chin at 1 week, patients selected an average of 3 words during both assessments but by 6 months, the average had decreased to 1 for the spontaneous and 2 for the evoked assessments of sensation. 


\section{PATIENTS' SELECTION OF WORDS WITHIN CATEGORIES OF INTRUSIVENESS}

Data from the chin are used to illustrate the percentages of patients who selected each word to describe the altered sensation at each of the 4 appointment times (Table 4). At 1 week after surgery some words were chosen by almost all patients. For example, $97 \%$ of the patients reported the sensation of "numb" (hypoesthesia category) during the spontaneous or evoked assessment of sensation on the chin. In contrast, the sensation of "wet" (also in the hypoesthesia category) was selected by only $2 \%$ of the patients. Similar differences for the report of numb versus wet were observed for all 4 sites, although the percentages were lower for the midface sites because fewer patients ( $42 \%)$ had maxillary surgery. "Rubbery" and "swollen" were commonly chosen from the hypoesthesia category for all 4 sites. The word "swollen" was selected more frequently than "rubbery" at 1 week after surgery, but "rubbery" was selected more frequently at 1,3 , and 6 months after surgery.

Similar to the hypoesthesia category, paresthesia was characterized largely by 3 words: "tingling," "tickling," and "itching." The word "twitching" was selected almost as often as "itching." Over 50\% of the patients reported "tingling" during either the spontaneous or the evoked assessment of sensation on the chin at every appointment time (Table 4). A similar finding was made for the lower vermilion, but not for the upper vermilion or upper lip.

The 3 most commonly chosen words in the dysesthesia category were "tender," "pricking," and "burning." Compared with the hypoesthesia and paresthesia categories, the 3 most commonly chosen words were less useful in characterizing dysesthesia. That is, many of the other words were selected almost as frequently. For example, the word "sore" was selected during either the spontaneous or evoked assessment of sensation on the chin by $15 \%$ of the patients at week 1 after surgery, approximating the $17 \%$ of patients who selected "tender," the most commonly chosen word (Table 4). However, "sore" was chosen by only $7 \%, 2 \%$, and $1 \%$ of the patients at 1,3 , and 6 months, respectively. It is plausible that "sore" better characterized the altered sensation from acute soft tissue trauma; whereas, "tender" better characterized inflammatory or neuropathic sequelae of the surgery.

Interestingly, only 9\% of the patients reported "pain" on the chin at 1 week after surgery. At other appointment times and for other sites, the percentages of patients who reported pain were lower than 9\%. This indicates that most patients did not consider their altered facial sensation as "painful," even though it had an unnatural, and in some cases an unpleasant, character.

\section{EXTENT OF RECOVERY AFTER SURGERY}

Most patients had not recovered normal sensation at 1 or more sites on the face by 6 months after surgery. To illustrate, $98 \%$ of the subjects before surgery reported normal sensation, ie, they did not indicate spontaneous or evoked altered sensation at any site evaluated on the face. However, at 1 week after surgery less than $2 \%$ of the patients reported normal sensation (Fig 1). Even after 6 months, only $37 \%$ and $16 \%$ of patients reported normal sensation at all 4 sites evaluated during the spontaneous and evoked assessments, respectively.

\section{RESOLUTION OF EVOKED VERSUS SPONTANEOUS ALTERATIONS IN SENSATION}

At each of the 4 sites, more patients reported a qualitative alteration in sensation at 6 months after surgery by the evoked assessment than by the spontaneous assessment of sensation. For example, for the chin, $81 \%$ of patients selected at least 1 word to describe sensory alteration during the evoked assessment; whereas, only $58 \%$ selected at least 1 word during the spontaneous assessment (Table 5). Moreover, 66\% of the patients were classified in the paresthesia and dysesthesia categories by the evoked assessment of sensation; whereas, only $47 \%$ were classified as such by the spontaneous assessment. 


\section{COURSE OF RECOVERY AFTER SURGERY}

Across all sites, there was a statistically significant inverse correlation between time after surgery and the maximum intrusiveness of the words chosen $\left(I A S_{\max }\right)$ during both the spontaneous (IAS max spont,$P<.001)$ and evoked $\left(I A S_{\text {max evok }}, P<.001\right)$ assessments of sensation. The percentages of patients who reported no alteration at any site on the face increased over time (Fig 1) while the percentages of patients who selected 1 or more words from the dysesthesia category decreased, at least for the assessment of spontaneous sensations (Fig 2, bottom). These trends were observed for each individual site. In contrast, the percentage of patients in the hypoesthesia and paresthesia category remained about the same (Fig 2, top and middle). Although the tendency was for the intrusiveness of the words selected to decrease over time, approximately $32 \%$ of the patients did not change categories, or the intrusiveness of the word(s) they chose worsened.

\section{RECOVERY OF PATIENTS WHO UNDERWENT DIFFERENT TYPES OF SURGERY, WITH AND WITHOUT GENIOPLASTY}

The percentage of patients classified in each intrusiveness category for the upper lip (Fig 3) and the chin (Fig 4) at 1 week and 6 months is illustrated in the figures for the 4 surgical groups of patients characterized in Table 2: BSSO only with and without genioplasty, BSSO + LFI surgery with and without genioplasty. It is apparent from Figure 3 that, as expected, patients who had a maxillary procedure were more likely to experience altered sensation in the distribution of the maxillary nerve than those who had a mandibular procedure only. Moreover, consistent with the percentages shown in Figure 3, the average intrusiveness (average $I A S_{\text {spon }}$ and $I A S_{\text {evok }}$ ) for the upper lip and vermilion differed among the 4 surgical groups at both 1 week and 6 months. The average level of intrusiveness was significantly greater than 0 , implying altered sensation, only for patients who underwent maxillary surgery. For these patients the mean values were similar for the upper lip and upper vermilion, and did not differ for patients with or without a genioplasty. As suggested by Figure 3, most patients recovered normal sensation on the upper lip by 6 months after surgery (approximately 93\% and on the upper vermilion, 95\%). The percentages are high, in part, because $58 \%$ of the patients did not have maxillary surgery.

In contrast to recovery on the upper lip and vermilion, recovery was particularly poor on the chin with $81 \%$ of the patients selecting words in 1 or more categories to describe evoked sensations at 6 months after surgery (Fig 4). At this time during recovery, the percentages of patients classified in the different categories of altered sensation were fairly similar for patients in the different surgical groups, all of whom had received BSSO (Fig 4).

\section{EFFECT OF TYPE OF SURGERY ON PATIENTS' SELECTION OF WORDS}

Differences among the surgical groups became evident upon analysis of the most common words that were chosen by patients at 1 or more of the postsurgical appointments. On the chin and lower vermilion, word usage was remarkably similar for patients who had BSSO only (without a genioplasty) or BSSO + LFI (without a genioplasty). For example, on the chin 100\% of the patients who had BSSO only reported "numb" during either the spontaneous or evoked assessment of sensation, compared with $98 \%$ of the patients who had BSSO + LFI surgery. Remarkably similar agreement was found also for "tingling" ( $87 \%$ vs $85 \%)$ and "tender" $(25 \%$ vs 23\%). Similar findings were observed for the lower vermilion. These findings indicate that the addition of maxillary surgery to BSSO did not significantly affect the way patients perceived and reported altered sensation on their lower vermilion and chin.

In contrast, and as expected, those patients who did and did not have a maxillary procedure differed greatly in their word usage for the upper vermilion and upper lip. Unexpectedly, however, $11 \%$ of the patients who had surgery on the mandible only reported that the upper 
lip was "numb" at 1 or more appointments. In addition, $8 \%$ of the patients reported tingling and $8 \%$ reported tickling sensations.

Maxillary surgery in general was less likely to result in altered sensation than mandibular surgery. For example, "numb" and "tingling" were reported on the upper lip during the spontaneous or evoked assessment of sensation by $78 \%$ and $58 \%$ of the BSSO + LFI patients, respectively. In comparison, "numb" and "tingling" were reported on the chin by $98 \%$ and $85 \%$ of the same patients. In contrast to use of words from the hypoesthesia and paresthesia categories, words from the dysesthesia category were used with about the same frequency on mid-face and lower face sites. For example, "tender," "prickling," and "burning" were reported on the upper vermilion during the spontaneous or evoked assessment of sensation by $20 \%$, $15 \%$, and $5 \%$ of the patients, respectively. Similarly, the 3 words were reported on the lower vermilion by $20 \%, 15 \%$, and $10 \%$ of the patients. These findings suggested that hypoesthesia and paresthesia, but not dysesthesia, were less of a problem on the midface than on the lower face after BSSO + LFI surgery.

There was a tendency for patients who had a genioplasty to more frequently choose descriptors for the lower face that are reflective of soft tissue trauma ("swollen" and "tender") and inflammation ("tender" and "burning") than were patients who did not have a genioplasty. For example, at some time after surgery "swollen" was reported on the chin by $60 \%$ of the patients who had genioplasty, compared with $41 \%$ of the patients who did not have genioplasty. Burning was reported on the chin and lower vermilion by $22 \%$ of the patients who had genioplasty, compared with $13 \%$ of those who did not.

\section{Discussion}

The first step in the "working guidelines" for the diagnosis of sensory disorders involves a patient interview with an emphasis on the patient's qualitative description of the sensory complaint. ${ }^{24}$ The current findings make evident that patients' selection of words differentiates individuals who experience only a simple loss in sensation (ie, present negative symptoms), those who experience active sensations that are not normally present (ie, positive symptoms), and those whose active sensations are uncomfortable or painful. Use of a clinically recommended, standardized list of words made it possible to detect subtle differences in subjective reports during the course of early postoperative recovery, between spontaneously occurring and evoked altered sensations, and between patients who underwent different surgical procedures. The analysis was motivated, in part, by the study of Gregg, ${ }^{25}$ who showed the utility of the words selected by patients with neuropathic pain to describe their altered sensation. By clinical examination and sensory testing, the symptoms of the patients, all candidates for trigeminal nerve exploration/repair surgery, were classified into 4 symptom components: anesthesia dolorosa, sympathetically mediated pain, hyperalgesia, or hyperpathia. The patients also completed a modified form of the McGill Pain Questionnaire. It was found that largely nonoverlapping categories of word descriptors from the McGill Pain Questionnaire were associated with the different symptom components. For example, hyperpathia was associated with words such as sore, tender, and aching. The prognosis for recovery differed among patients with different symptom components suggesting that the words chosen by patients to describe altered sensation following injury to the trigeminal nerve had both diagnostic and prognostic value.

Only a few studies have attempted to characterize differences among patients experiencing altered sensation based on their selection of words from a standardized list. Other differences make it difficult to compare the results of the present study with those reported in the literature. For example, using a standardized list of 14 words, Upton et al9 obtained word selections from orthognathic surgery patients and patients after third molar extraction, a procedure which 
entails a lower risk of neurosensory impairment than orthognathic surgery. As expected, the percentages of patients in the present study classified in the paresthesia and dysesthesia categories at 6 months after surgery were higher than those suggested in Upton et al. ${ }^{9}$ In the present study, approximately two thirds of the patients at 6 months after surgery reported positive symptoms, in addition to losses in sensation. Moreover, roughly one third of the patients selected words at 6 months that were no less intrusive than the words selected at earlier postsurgical appointments, and in some case the words came from more intrusive categories. In the Upton et al study, the most commonly selected words during the first 6 months following surgery were numbness (approximately $60 \%$ of the patients), tingling (39\%), and prickling (26\%) - words that were among the most commonly chosen in the present study from the hypoesthesia, paresthesia, and dysesthesia categories, respectively. The present study also sought and identified subtle changes in sensation during recovery, suggested by changes in the frequency of use of individual words. This is illustrated by the change from 1 week to 6 months in the relative use of "swollen" versus "rubbery," and of "sore" versus "tender." Such changes indicate that the altered sensation reported by patients is caused by acute tissue trauma, inflammation, and healing as well as nerve injury. Further work is needed to determine if and when the 2 components of altered facial sensation can be distinguished.

Between 1 and 6 months, pain was reported by approximately $13 \%$ of the patients in the Upton et al study. ${ }^{9}$ Similar to the present study, the percentage of patients selecting words to describe altered sensation decreased with time, eg, pain was reported by only $5 \%$ of patients at $\geq 6$ months after surgery. Words describing unpleasant and uncomfortable sensations were chosen much more frequently than the word "pain" in the current study. This indicates that symptoms of neuropathic pain may be missed in the evaluation of patients if they are only given the option to rate the magnitude of pain, or report its presence. Of course, patients who select words from the dysesthesia category during the early postoperative period are not necessarily likely to develop neuropathic pain. The 6-month assessment time is beyond that expected for peripheral receptor and nerve trunk recovery after trigeminal nerve injury, but the central nervous system may require a year or more before it reaches adaptive normalcy. ${ }^{28}$ The subjects in this study will be followed until 2 years postsurgery and it is plausible that continued assessment of the words chosen, particularly for those whose immediate postoperative intrusiveness level did not improve during the first 6 months, may reveal patterns that predict poor long-term recovery and disabling sensory disorders.

\section{EFFECT OF TYPE OF SURGERY}

At 6 months, there is generally less sensory alteration on the midface than on the lower face in patients who undergo both mandibular and maxillary surgery. This is consistent with our previous finding that "2-jaw" patients are more likely to report less altered sensation on the upper lip than on the lower lip and chin at 6 weeks after surgery, and that pinprick and 2-point sensitivity are less impaired on the upper lip in these patients. ${ }^{3,6,23}$ The entire extracranial inferior alveolar nerve is mostly exposed with BSSO in every surgery, giving multiple opportunities for trauma. Only the terminal infraorbital nerve, outside the maxilla, is exposed during LFI osteotomies and this does not always occur.

Patients who undergo genioplasty experience more dysesthesia than patients who do not have a genioplasty in the short term after surgery. This finding extends our previous work and that of others which show that genioplasty entails an additional loss in sensory function beyond that associated with BSSO or LFI osteotomies. $4^{-} 6$ For example, Essick et $\mathrm{al}^{6}$ found that sensitivity to pinprick was impaired to a greater extent at 6 weeks after surgery for patients who underwent a genioplasty procedure. As would be anticipated, the words chosen by the patients in the present study to describe sensation on the chin were largely reflective of soft tissue injury and inflammation (ie, "swollen," "tender," and "burning"). Importantly, the 
present study found that altered sensation is roughly comparable for patients with and without genioplasty by 6 months after surgery.

\section{RELATION OF OBJECTIVE MEASURES OF NEUROSENSORY FUNCTION AND PATIENTS' SELECTION OF WORDS}

Anecdotal observations suggest that patients who report qualitatively different altered sensations may represent distinct subgroups of patients, whose objective neurosensory testing results should not be combined in clinical studies. Pooling sensory threshold values from patients in different subgroups may inflate estimates of among-subject variability, thereby decreasing the power of statistical analyses, and masking true differences among patients. ${ }^{14}$, ${ }^{22,28}$ For example, Cunningham et $\mathrm{al}^{22}$ averaged measures of patients' performance on tests of tactile detection and of brush-stroke discrimination, but noted that patients who reported tingling had lower thresholds at 6 months after surgery than presurgically. Patients who reported only numbness tended to have higher thresholds postsurgically. A similar situation was identified by Essick et al, ${ }^{14}$ who showed that analysis of the data from trigeminal nerve injured patients revealed no deficit in cold pain detection. However, when the patients who reported increased sensitivity to cold were eliminated, reanalysis showed the presence of substantial cold hypoalgesia for the remaining group of individuals, ie, lower temperatures were required for cold pain perception.

The presence of paresthesia (as in Cunningham et al22) or discomfort (as in Essick et al14) does not necessarily suggest that sensitivity to tactile or thermal stimuli is increased. In fact, post-traumatic neuropathic pain by definition requires that there be nerve injury and sensory loss,29 particularly involving unmyelinated or small diameter afferents.17,18,30 Thus, there is no simple obligatory relation between the intrusiveness of the words chosen to describe altered sensation and increased sensitivity upon objective sensory testing. The percentage of patients who reported evoked alterations in sensation were substantially higher at 6 months after surgery than the percentage who reported spontaneous alterations, yet the words chosen were largely similar. Abnormal evoked sensations, unlike spontaneous ones, suggest stimulation of suprasensitive regenerating nerve fibers or sensitization within the peripheral or central nervous system. Clearly, additional work is needed to clarify the relationships between the quality of the evoked and spontaneous alterations in sensation reported by patients and the polarity of abnormal results from sensory testing (ie, increased versus decreased threshold values).

\section{Acknowledgments}

The authors thank Colleen Ellett Farmer, Harold Jennings, Debora Price, Akshya Patel, and Atousa Safavi for their efforts in patient enrollment and data collection.

This project was supported in part by National Institutes of Health grant no. DE01367.

\section{References}

1. Westermark A, Bystedt $\mathrm{H}$, von Konow L. Inferior alveolar nerve function after sagittal split osteotomy of the mandible: Correlation with degree of intraoperative nerve encounter and other variables in 496 operations. Br J Oral Maxillofac Surg 1998;36:429. [PubMed: 9881784]

2. Zuniga JR, Chen N. Nerve injuries: Considerations in orthognathic surgery. Oral Maxillofac Surg Knowledge Update 1995;1:43.

3. Karas ND, Boyd SB, Sinn DP. Recovery of neurosensory function following orthognathic surgery. J Oral Maxillofac Surg 1990;48:124. [PubMed: 2299455]

4. Posnick JC, Al-Quattan MM, Stepner NM. Alteration in facial sensibility in adolescents following sagittal split and chin osteotomies of the mandible. Plast Reconstr Surg 1996;97:920. [PubMed: 8618994] 
5. Nishioka GJ, Zysset JK, Van Sickels JE. Neurosensory disturbance with rigid fixation of the bilateral sagittal split osteotomy. J Oral Maxillofac Surg 1987;45:20. [PubMed: 3467034]

6. Essick G, Austin S, Phillips C, et al. Short term neurosensory alterations in orthognathic surgery patients. Oral Maxillofac Surg Clin North Am 2001;13:295.

7. Blakey GH, Zuniga JR. Lingual nerve injury associated with superior border wire fixation. Int J Adult Orthognath Surg 1992;7:115.

8. Tyndall DA, Gregg JM, Hanker JS. Evaluation of peripheral nerve regeneration following crushing or transection injuries. J Oral Maxillofac Surg 1984;42:314. [PubMed: 6585504]

9. Upton LG, Rajvanakarn M, Hayward JR. Evaluation of the regenerative capacity of the inferior alveolar nerve following surgical trauma. J Oral Maxillofac Surg 1987;45:212. [PubMed: 3469360]

10. Sandstedt P, Sorensen S. Neurosensory disturbances of the trigeminal nerve: A long term follow-up of traumatic injuries. J Oral Maxillofac Surg 1995;53:498. [PubMed: 7722715]

11. Coghlan KM, Irvine GH. Neurological damage after sagittal split osteotomy. Int J Oral Maxillofac Surg 1986;15:369. [PubMed: 3091714]

12. Essick G. Psychophysical assessment of patients with posttraumatic neuropathic trigeminal pain. J Orofac Pain 2004;18:345. [PubMed: 15636019]

13. Essick GK. Comprehensive clinical evaluation of perioral sensory function. Oral Maxillofac Surg Clin North Am 1992;4:503.

14. Essick GK, Patel S, Trulsson M. Mechano- and thermosensory changes across the border of impaired sensitivity to pinprick after mandibular nerve injury. J Oral Maxillofac Surg 2002;60:1250. [PubMed: 12420257]

15. Teerijoki-Oksa T, Jaaskelainen S, Forssell K, et al. An evaluation of clinical and electrophysiological tests in nerve injury diagnosis after mandibular sagittal split osteotomy. Int J Oral Maxillofac Surg 2003;32:15. [PubMed: 12653227]

16. Teerijoki-Oksa T, Jaaskelainen SK, Forssell K, et al. Recovery of nerve injury after mandibular sagittal split osteotomy. Diagnostic value of clinical and electrophysiologic tests in the follow-up. Int J Oral Maxillofac Surg 2004;33:134. [PubMed: 15050068]

17. Jaaskelainen SK. The utility of clinical neurophysiological and quantitative sensory testing for trigeminal neuropathy. J Orofacial Pain 2004;18:355. [PubMed: 15636020]

18. Jaaskelainen SK, Teerijoki-Oksa T, Virtanen A, et al. Sensory regeneration following intraoperatively verified trigeminal nerve injury. Neurology 2004;62:1951. [PubMed: 15184595]

19. Harvey WS, Phillips CL, Essick GK. Neurosensory impairment and patient perception of recovery following orthognathic surgery. J Dent Res 2001;80(Special Issue):187.

20. Westermark A, Bystedt H, von Konow LL. Patients' evaluation of the final result of sagittal split osteotomy: Is it influenced by impaired sensitivity of the lower lip and chin? Int Adult Orthod Orthognath Surg 1999;14:135.

21. Westermark A, Englesson L, Bongenhielm U. Neurosensory function after sagittal split osteotomy of the mandible: A comparison between subjective evaluation and objective assessment. Int J Adult Orthod Orthognath Surg 1999;14:268.

22. Cunningham LL, Tiner BD, Clark GM, et al. A comparison of questionnaire versus monofilament assessment of neurosensory deficit. J Oral Maxillofac Surg 1996;54:454. [PubMed: 8600262]

23. Chen N, Neal CE, Lingenbrink P, et al. Neurosensory changes following orthognathic surgery. Int $J$ Adult Orthognath Surg 1999;14:259.

24. Zuniga JR, Essick GK. A contemporary approach to the clinical evaluation of trigeminal nerve injuries. Oral Maxillofac Surg Clin North Am 1992;4:353.

25. Gregg JM. Studies of traumatic neuralgias in the maxillofacial region: Surgical pathology and neural mechanisms. J Oral Maxillofac Surg 1990;48:228. [PubMed: 2303934]

26. Gregg JM. Nonsurgical management of traumatic trigeminal neuralgias and sensory neuropathies. Oral Maxillofac Surg Clin North Am 1992;4:375.

27. Meyer RA, Rath EM. Sensory rehabilitation after trigeminal nerve injury or nerve repair. Oral Maxillofac Surg Clin North Am 2001;13:365.

28. Gregg JM. Discussion: A comparison of questionnaire versus monofilament assessment of neurosensory deficit. J Oral Maxillofac Surg 1996;54:459. 
29. Burchiel KJ. Trigeminal neuropathic pain. Acta Neurochir 1993;58:145. (Wien).

30. Cruccu G, Anand P, Attal N, et al. EFNS guidelines on neuropathic pain assessment. Eur J Neurol 2004;11:153. [PubMed: 15009162] 


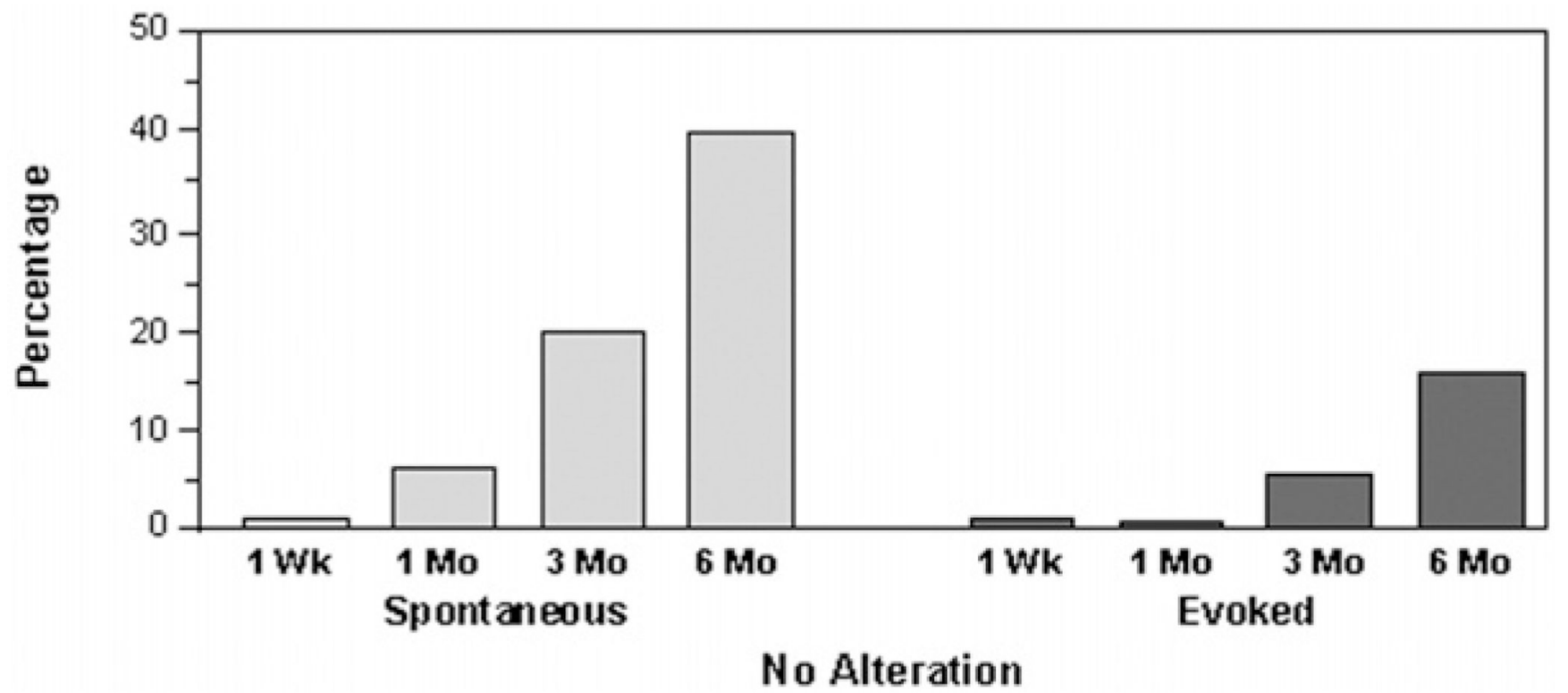

Figure 1.

Percentage of patients who reported no altered sensation anywhere on the face at each of the postsurgery appointments. 

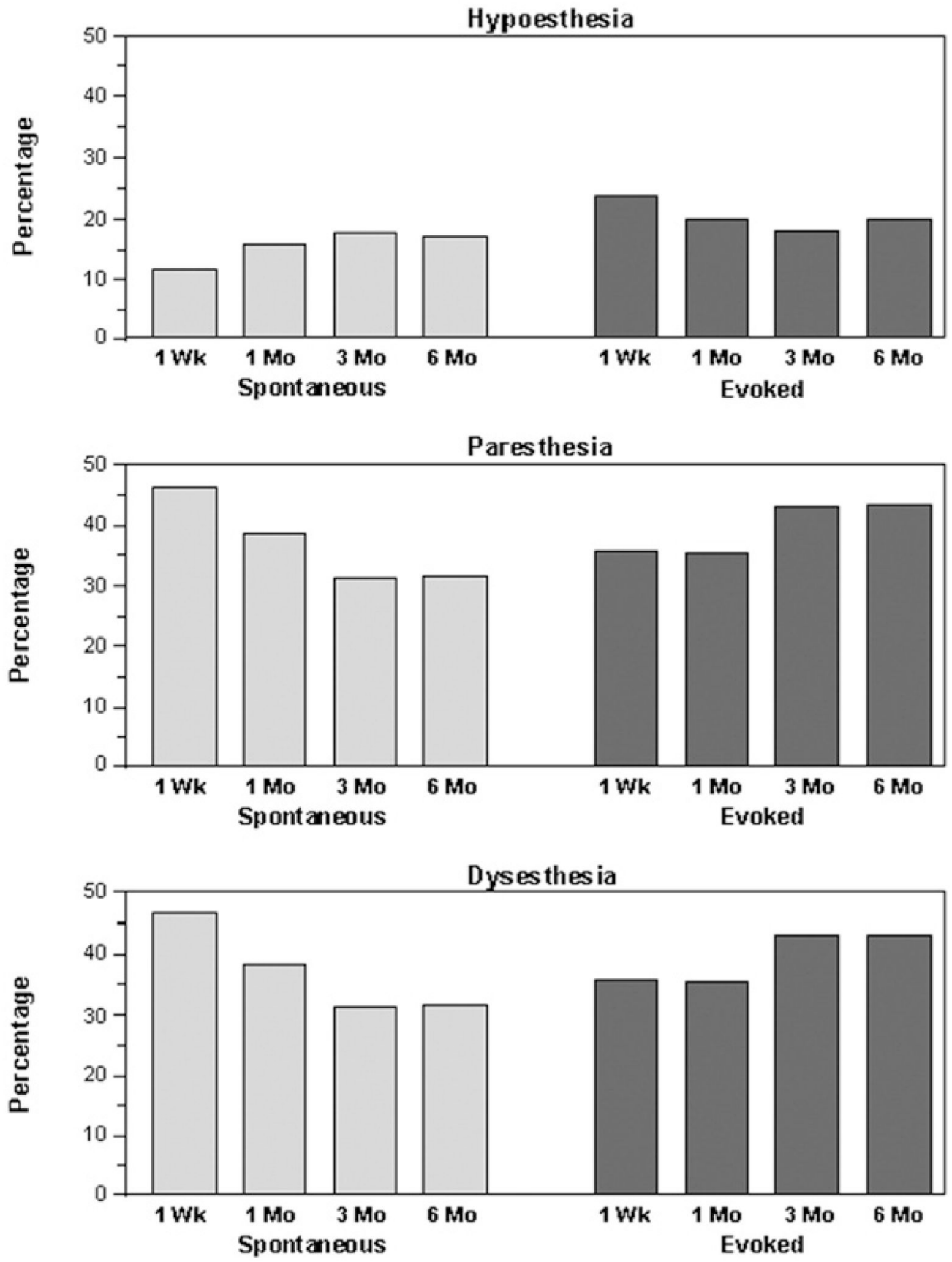

Figure 2.

Percentage of patients classified into one of the altered sensation categories (hypoesthesia, paresthesia, dysesthesia) at each of the postsurgery appointments according to the most intrusive word(s) chosen to describe the altered sensation anywhere on the face. 

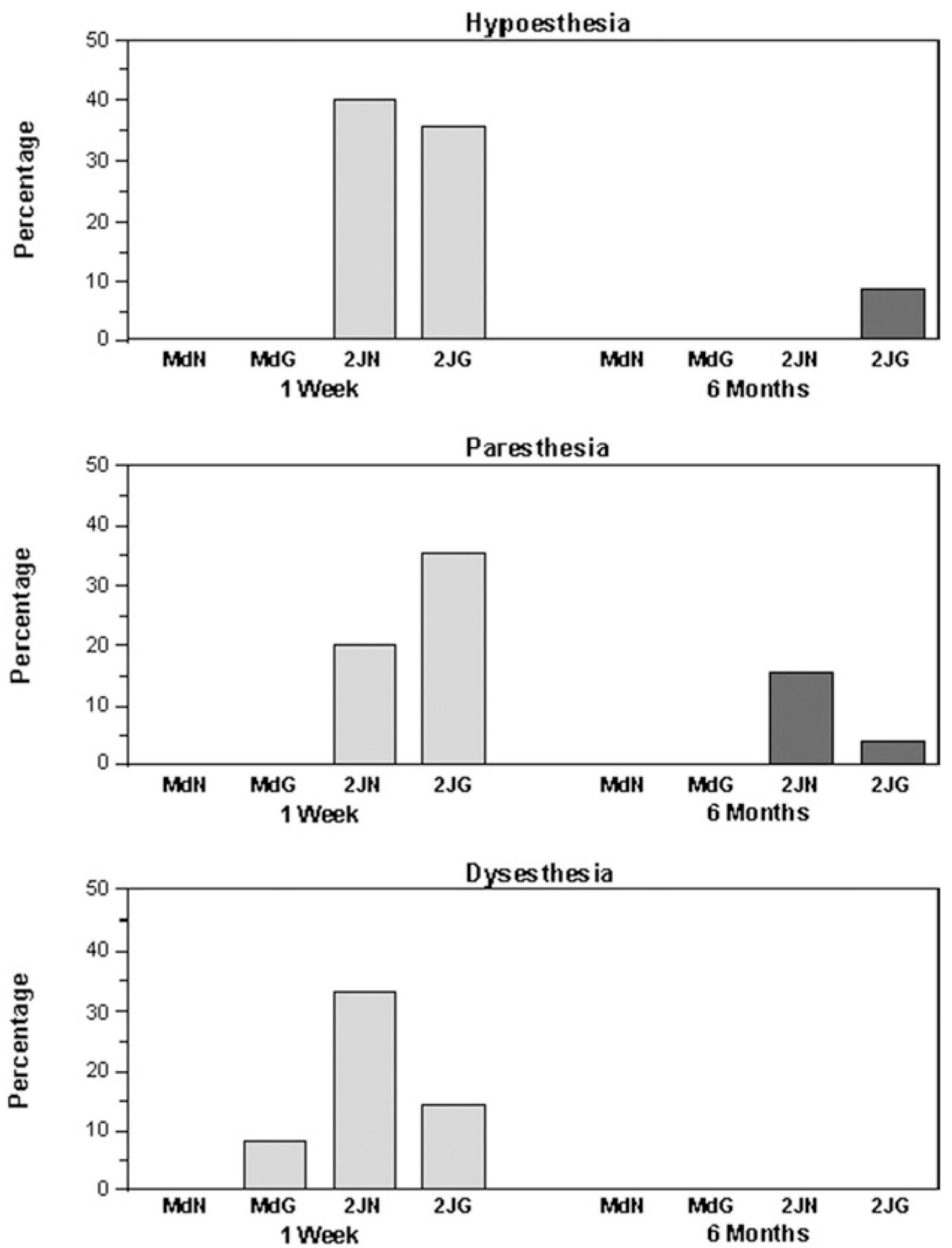

Figure 3.

Using only words chosen to describe the upper lip during evoked testing, percentage of patients classified into one of the altered sensation categories (hypoesthesia, paresthesia, dysesthesia) at 1 week and 6 months separately for the 4 surgical groups: BSSO only, no genioplasty (MdN); BSSO with genioplasty (MdG); 2-jaw, no genioplasty (2JN); 2-jaw with genioplasty (2JG). 

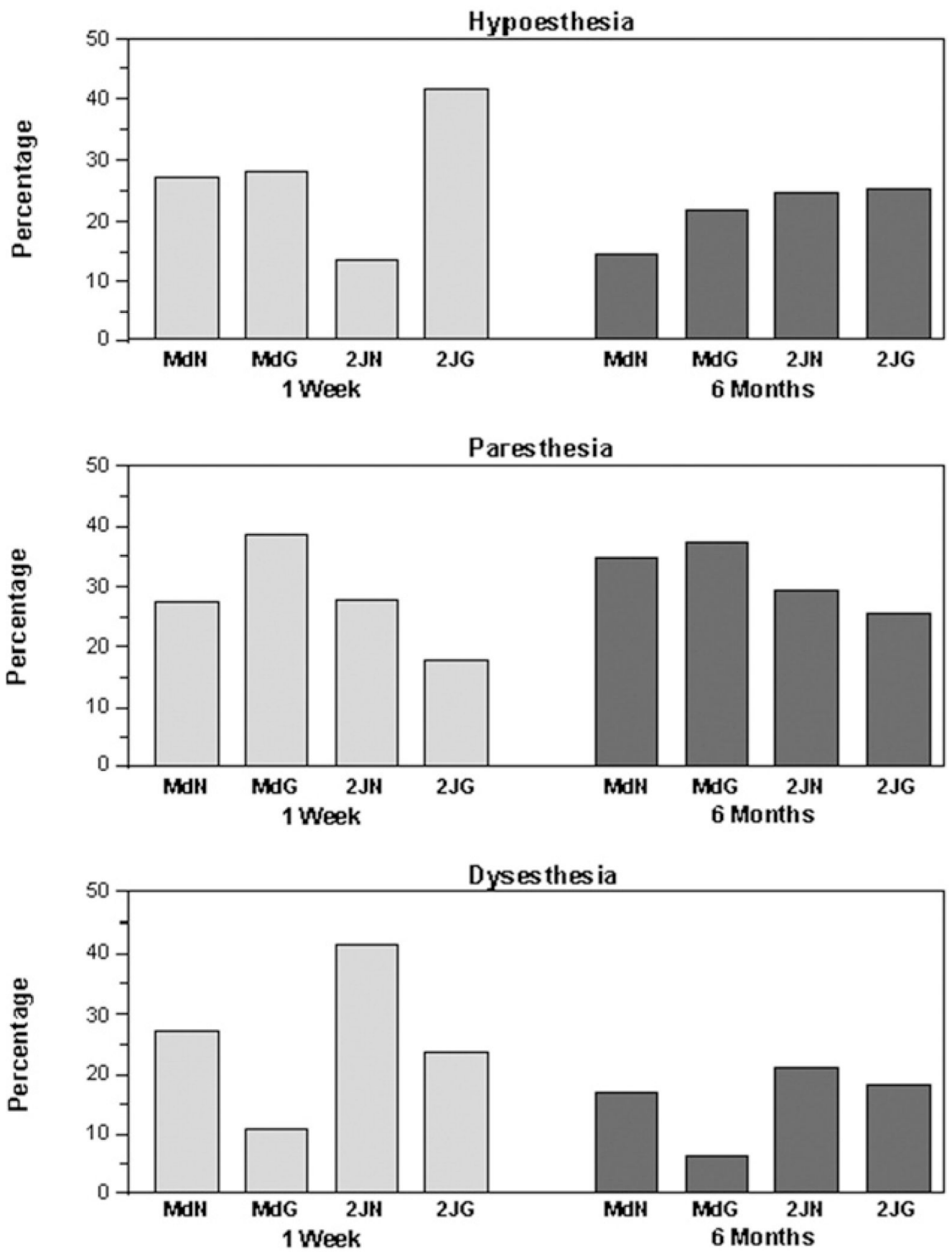

Figure 4.

Using only words chosen to describe the chin during evoked testing, percentage of patients classified into one of the altered sensation categories (hypoesthesia, paresthesia, dysesthesia) at 1 week and 6 months separately for the 4 surgical groups: BSSO only, no genioplasty (MdN); BSSO with genioplasty (MdG); 2-jaw, no genioplasty (2JN); 2-jaw with genioplasty (2JG). 
Table 1

INCLUSION AND EXCLUSION CRITERIA FOR ENROLLMENT IN THE SENSORY RETRAINING CLINICAL TRIAL

Inclusion criteria

1 Have a developmental dentofacial disharmony

2 Be 13 to 50 years of age

3 Be scheduled to receive a bilateral sagittal split either by mandibular osteotomy only or combined mandibular/maxillary surgery

Exclusion criteria

1 Have a congenital anomaly or acute trauma

2 Have had previous facial surgery

3 Are pregnant at baseline

4 Do not have the ability to follow written English instructions

5 Are unwilling to sign informed consent

6 Report a moderate level of discomfort or problem caused by altered sensation of numbness or unusual feeling on the face at baseline

7 Report no altered sensation at 1 week postsurgery

8 Have a medical condition associated with systemic neuropathy (eg, diabetes, hypertension, kidney problems) 


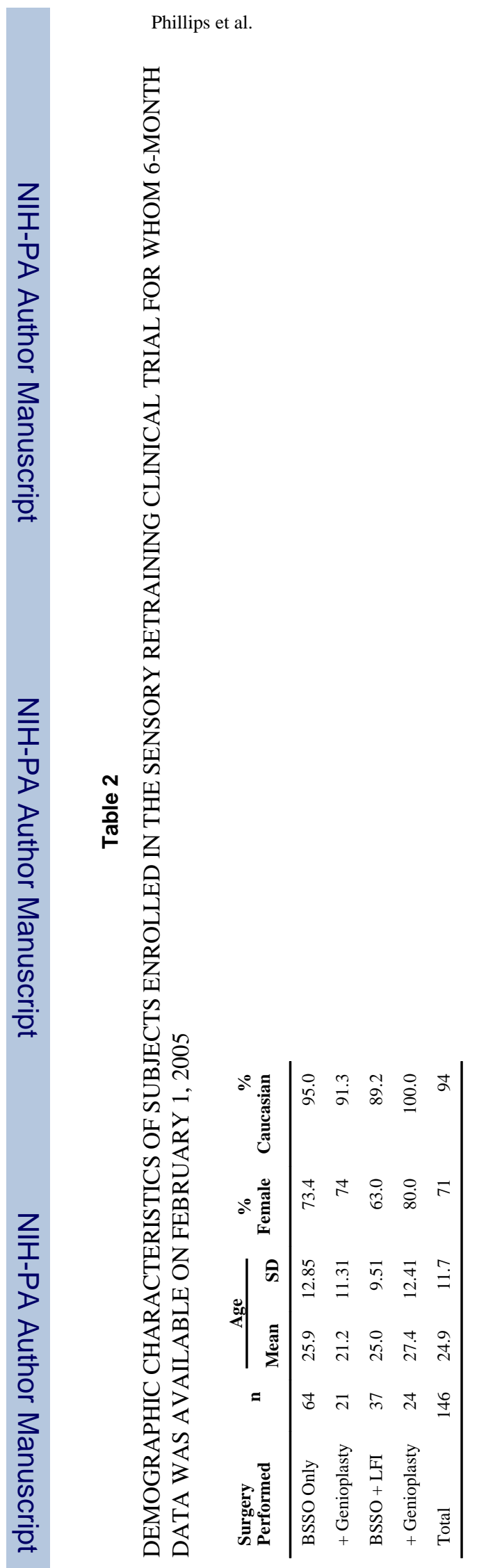

J Oral Maxillofac Surg. Author manuscript; available in PMC 2010 May 13. 


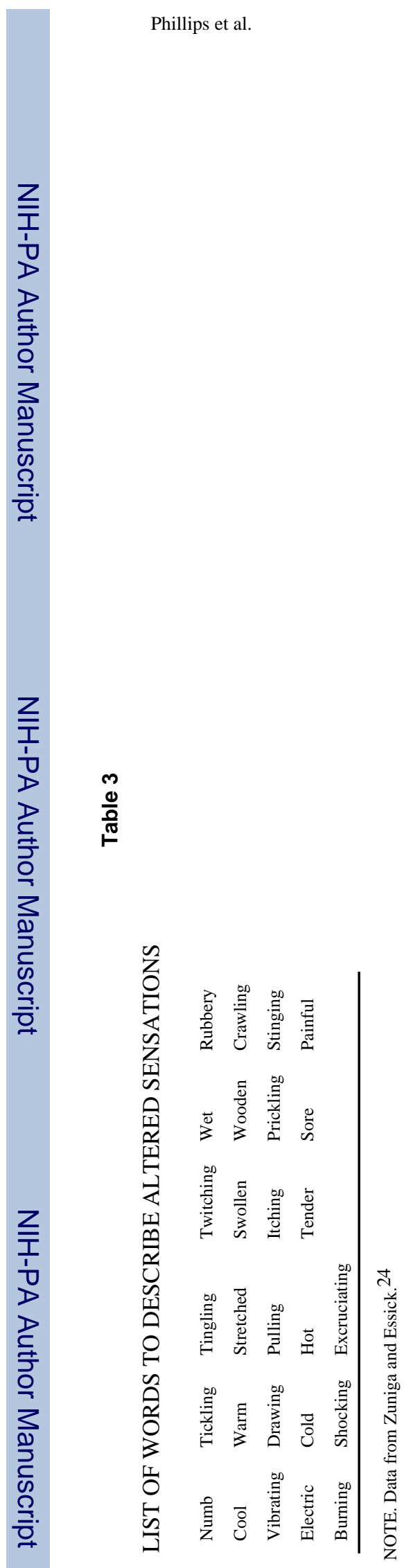

J Oral Maxillofac Surg. Author manuscript; available in PMC 2010 May 13. 
Table 4

PERCENTAGE OF PATIENTS ( $\mathrm{N}=146)$ WHO CHOSE EACH WORD TO DESCRIBE ALTERED SENSATION ON THE CHIN EXPERIENCED SPONTANEOUSLY OR EVOKED BY FACIAL EXPRESSION OR LIGHT TOUGH

\begin{tabular}{|c|c|c|c|c|}
\hline & \multicolumn{4}{|c|}{$\begin{array}{c}\text { Spontaneous or } \\
\text { Evoked Altered Sensation } \\
\end{array}$} \\
\hline & $\begin{array}{c}1 \text { week } \\
(\%)\end{array}$ & 1 month & 3 month & 6 month \\
\hline \multicolumn{5}{|l|}{ Hypoesthesia } \\
\hline Numb & 97 & 91 & 72 & 61 \\
\hline Rubbery & 31 & 30 & 23 & 21 \\
\hline Swollen & 52 & 23 & 9 & 10 \\
\hline Cool & 11 & 11 & 14 & 9 \\
\hline Stretched & 8 & 6 & 6 & 4 \\
\hline Warm & 12 & 6 & 6 & 3 \\
\hline Wooden & 9 & 7 & 4 & 4 \\
\hline Wet & 2 & 2 & 3 & 3 \\
\hline \multicolumn{5}{|l|}{ Paresthesia } \\
\hline Tingling & 67 & 60 & 65 & 54 \\
\hline Tickling & 35 & 34 & 35 & 26 \\
\hline Itching & 25 & 32 & 25 & 7 \\
\hline Twitching & 14 & 14 & 9 & 4 \\
\hline Vibrating & 6 & 5 & 8 & 5 \\
\hline Pulling & 3 & 5 & 5 & 4 \\
\hline Crawling & 6 & 8 & 4 & 4 \\
\hline Drawing & 2 & 3 & 3 & 4 \\
\hline \multicolumn{5}{|l|}{ Dysesthesia } \\
\hline Tender & 17 & 11 & 15 & 6 \\
\hline Pricking & 13 & 14 & 12 & 9 \\
\hline Burning & 8 & 8 & 6 & 5 \\
\hline Stinging & 7 & 8 & 5 & 5 \\
\hline Electric & 2 & 8 & 6 & 4 \\
\hline Sore & 15 & 7 & 2 & 1 \\
\hline Painful & 9 & 5 & 7 & 3 \\
\hline Hot & 3 & 1 & 1 & 1 \\
\hline Cold & 3 & 2 & 6 & 4 \\
\hline Shocking & 3 & 5 & 3 & 2 \\
\hline
\end{tabular}

NOTE. Data were collected at 1 week, 1 month, 3 months, and 6 months after surgery. 


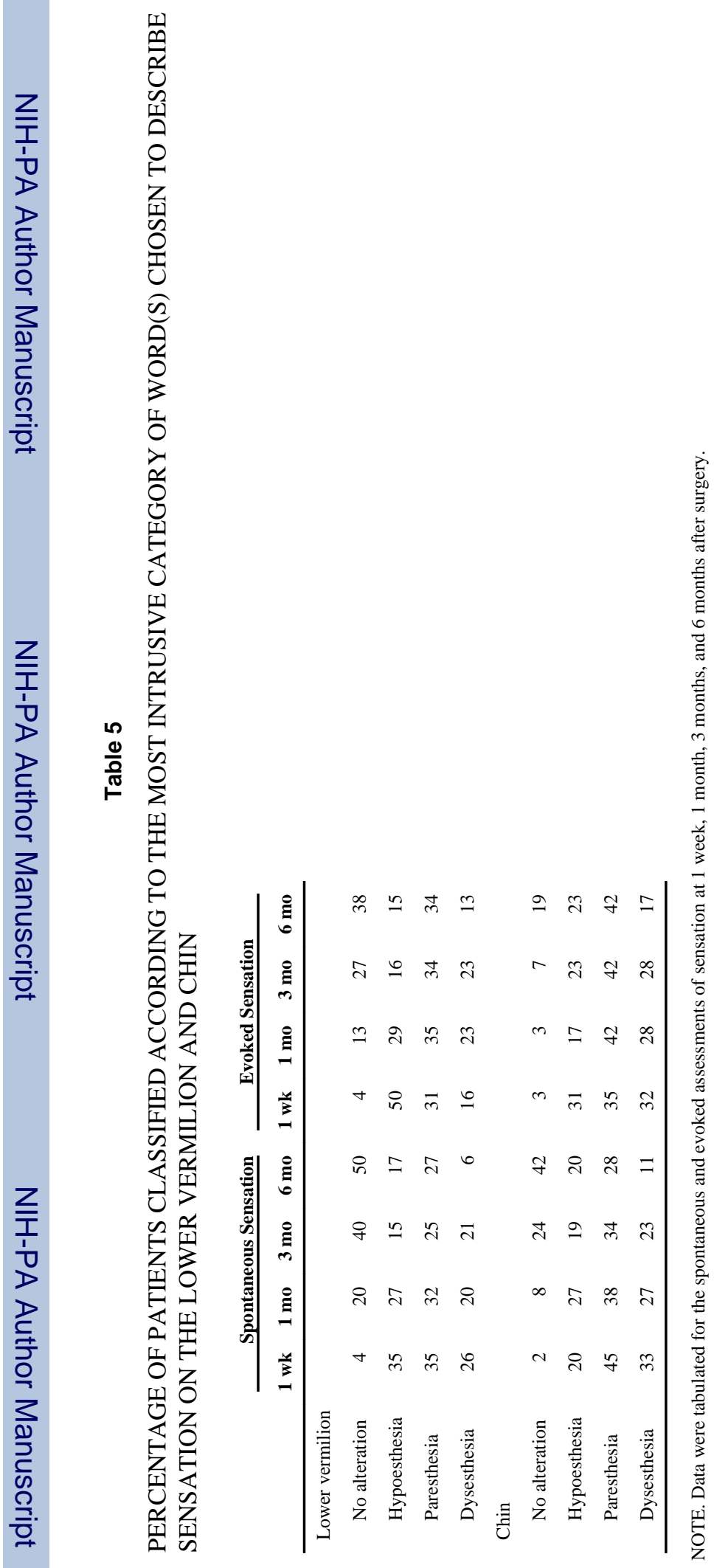

J Oral Maxillofac Surg. Author manuscript; available in PMC 2010 May 13. 